\title{
OTIMIZAÇÃO DO COZIMENTO DE FILÉ DE FRANGO EMPANADO EM FORNO DE INJEÇÃO DIRETA DE VAPOR ${ }^{1}$
}

\author{
Cristina LEONHARDT ${ }^{2}$, Helena Caminha Pereira dos SANTOS ${ }^{3}$,
}

Ligia Damasceno Ferreira MARCZAK ${ }^{4}$, Caciano Pelayo ZAPATA-NOREÑA ${ }^{5, *}$

\section{RESUMO}

Foi estudado o efeito da temperatura do ar $\left(205-215^{\circ} \mathrm{C}\right)$ e do tempo de cozimento $(3,5$ - 4,1 minutos), sobre a temperatura interna e o rendimento durante o cozimento do filé de frango empanado usando a metodologia de superfície de resposta, obtendo-se modelos estatísticos adequados. Verificou-se que as melhores condições de processo foram o emprego de temperaturas entre 206 e $209^{\circ} \mathrm{C}$ e tempos entre 2,5 e 3,6 minutos.

Palavras-chave: superficie de resposta; frango; cozimento; transferência de calor.

\section{SUMMARY}

OPTIMIZING OF IMPINGEMENT ON COOKING FILLET CHICKEN. The effect of air temperature $\left(205-215^{\circ} \mathrm{C}\right)$ and cooking time $(3.5-4.1$ minutes) on the internal temperature and the yield of fillet chicken were studied using response surface methodology. Satisfactory statistical models were obtained. The best conditions were temperatures between 206 and $209^{\circ} \mathrm{C}$ and times between 2.5 and 3.6 minutes.

Keywords: response surface; chicken; cooking; heat transfer.

\section{1-INTRODUÇÃO}

O atual estágio da tecnologia de processamento de carnes permite que muitos alimentos, que antes necessitavam de preparo doméstico, cheguem à casa do consumidor já prontos, por vezes, totalmente cozidos. No caso do frango uma das formas de apresentação é como filé empanado totalmente cozido, tendo como principal operação unitária a etapa de cozimento. O cozimento pode ser realizado em fornos de injeção direta ou indireta de vapor, radiação, microondas, etc., e a compreensão dos mecanismos de atuação do calor sobre o produto são pontos de grande importância para a seleção do melhor programa de cozimento, que deve atender às condições de segurança alimentar e ao mesmo tempo gerar um rendimento tal que a produção seja vantajosa para a indústria.

ANG \& HUANG [1] estudaram o efeito da temperatura mais alta atingida no centro de empadas, feitas a partir do músculo da perna de frango, na faixa de 60 a $85^{\circ} \mathrm{C}$ sobre a cor (medida no sistema Lab Hunter) da carne cozida em grelha. Eles verificaram que, quando se aumenta a temperatura a luminosidade (L) e o amarelo versus azul (b) aumentam; enquanto que o vermelho versus verde (a) diminui.

BERRY [2] verificou que empadas de carne de boi, cozidas numa grelha elétrica, contendo $20 \%$ de gordura

\footnotetext{
1. Recebido para publicação em 16/11/2002. Aceito para publicação em 05/11/2003 (000796).

2. UFRGS.

3. UFSC.

4. Departamento de Engenharia Química, Universidade Federal do Rio Grande do Sul.

5. Instituto de Ciência e Tecnologia de Alimentos, Universidade Federal do Rio Grande do Sul, Av. Bento Gonçalves 9500, CEP 91501-970, Porto Alegre, RS, Brasil, e-mail: czapatan@ufrgs.br.

*A quem a correspondência deve ser enviada.
}

na sua formulação, apresentaram maiores intensidade de aroma de carne, suculência, maciez e rendimentos que quando continha $4 \%$ de gordura.

SHERIDAN \& SHILTON [13] avaliaram o rendimento durante o cozimento da carne moída tipo hambúrguer usando um forno de radiação. Eles verificaram que a maior perda de água durante o aquecimento não obedecia ao modelo de transferência de massa de FICK, devido a sua estrutura capilar - porosa.

CHRISTENSEN, PURSLOW \& LARSEN [5] estudaram a influência da temperatura de cozimento sobre as propriedades mecânicas da carne e de seus principais componentes.

PEARSON \& GILLETT [9] mencionaram que o forno de injeção direta de vapor, tipo impingement, tem como característica principal os altos valores de coeficiente de película na transferência de calor quando comparados com fornos tradicionais de fluxo de ar estático ou os aquecidos por radiação.

O objetivo do presente trabalho foi avaliar através da metodologia de superfície de resposta, a influência do tempo de cozimento e temperatura do processo na temperatura interna final e rendimento do filé de frango empanado.

\section{2 - MATERIAIS E MÉTODOS}

Foi utilizado filé de frango empanado e para o cozimento foi empregado um forno de injeção direta de vapor, tipo impingement.

O rendimento do produto, que nos indica a quantidade de massa não perdida ou evaporada durante o cozimento, foi calculado a partir da razão entre a massa dos filetes na saída e na entrada do forno [2]. A pesagem das massas foi feita empregando uma balança semianalítica, com precisão de 0,01g. 
A temperatura dos filetes imediatamente após o cozimento foi medida no centro geométrico do produto mediante o emprego de um termômetro tipo agulha, com precisão de $0,1^{\circ} \mathrm{C}$. De acordo com o recomendado por WÄHLBY, SKJÖLDEBRAND \& JUNKER [14], a temperatura máxima no centro, utilizada como resposta, foi àquela imediatamente anterior ao início do decréscimo da temperatura.

\section{1 - Desenho experimental}

Foram empregados dois fatores: temperatura e tempo de cozimento. Os níveis dos fatores testados foram: 205, 210 e $215^{\circ} \mathrm{C}$ para a temperatura de cozimento e 3.5; 3.8; e 4.1 minutos para o tempo de processo. Os experimentos foram realizados seguindo um planejamento fatorial $3^{2}$ com três repetições no ponto central. As respostas foram a temperatura interna no frango e o rendimento.

Foi empregado um desenho central composto rotacional de segunda ordem e os resultados foram analisados usando a metodologia de superfície de resposta. O polinômio de segundo grau empregado para o ajuste das variáveis de resposta foi:

$$
Y=\beta_{o}+\beta_{1} x_{1}+\beta_{2} x_{2}+\beta_{11} x_{1}^{2}+\beta_{22} x_{2}^{2}+\beta_{12} x_{1} x_{2}
$$

Onde $x_{1}$ e $x_{2}$ são o tempo e a temperatura do processo respectivamente, expressados em forma codificada; $\beta_{0}, \beta_{1}, \beta_{2}, \beta_{11}, \beta_{22}, \beta_{12}$, são os parâmetros a serem estimados; $Y$ é a variável de resposta (temperatura interna e rendimento do filé de frango empanado).

\section{2 - Análise estatística}

Foi empregado o pacote estatístico SAS [12] para as análises de regressão, de variância e canônico. Para a construção das linhas de contorno foi empregado o pacote MATLAB [7].

\section{3 - RESULTADOS E DISCUSSÃO}

Na Tabela 1 estão apresentados os resultados dos experimentos obtidos da combinação dos fatores de tempo e temperatura de processo, onde se observa que as temperaturas finais do produto estiveram na faixa de 79,6 e $88,5^{\circ} \mathrm{C}$. A respeito, RESURRECCION [11] sugere que os pontos finais de cozimento (grau de cozimento pronto) para os cortes de peito e sobrecoxa de frango são obtidos nas temperaturas de 71 e $82^{\circ} \mathrm{C}$, respectivamente.

Para o rendimento dos filés de frango frito obtiveram-se valores entre 96,7 e 98,6\%, sendo que as perdas são devidas basicamente à saída de água do produto por evaporação. A respeito, HULTIN [8] menciona que uma das conseqüências da desnaturação das proteínas musculares pelo calor é a diminuição da retenção de água. A desnaturação pelo calor e a desidratação superficial durante o cozimento origina a formação de uma capa superficial que tende a diminuir a taxa de perda de água. Quanto aos lipídios eles se fundem e as células do tecido adiposo se rompem ocasionando uma importante redistribuição das gorduras dentro do produto.

TABELA 1. Rendimento dos filés de frango frito

\begin{tabular}{ccccc}
\hline Tratamento & $\begin{array}{c}\text { Tempo } \\
\text { (minutos) }\end{array}$ & $\begin{array}{c}\text { Temperatura } \\
\left({ }^{\circ} \mathrm{C}\right)\end{array}$ & $\begin{array}{c}\text { Temperatura } \\
\text { interna }\left({ }^{\circ} \mathrm{C}\right)\end{array}$ & $\begin{array}{c}\text { Rendimento } \\
(\mathrm{g} / 100 \mathrm{~g})\end{array}$ \\
\hline 1 & 3,5 & 210 & 82,0 & 98,3 \\
2 & 4,1 & 210 & 88,5 & 97,4 \\
3 & 3,5 & 215 & 79,6 & 98,0 \\
4 & 4,1 & 215 & 87,6 & 96,7 \\
5 & 3,8 & 210 & 84,4 & 97,7 \\
6 & 3,8 & 215 & 81,5 & 97,8 \\
7 & 3,5 & 205 & 79,9 & 98,6 \\
8 & 4,1 & 205 & 81,5 & 97,7 \\
9 & 3,8 & 205 & 80,9 & 98,2 \\
10 & 3,8 & 210 & 79,8 & 97,9 \\
11 & 3,8 & 210 & 83,7 & 97,7 \\
\hline
\end{tabular}

CHEN, MARKS \& MURPHY [4] verificaram que o rendimento da carne de frango é afetado pela transferência de massa, principalmente sob a forma de transferência de água do produto para o exterior, que é diminuído pela formação de uma camada superficial com menor concentração de água do que no centro, camada que acaba por formar uma barreira para a continuidade da saída de água.

O cozimento rápido da carne, a coloração homogênea e os altos rendimentos obtidos (pouca perda de água) é característica desse tipo de forno [14].

\section{1 - Temperatura interna do frango}

Na Tabela 2, estão apresentados os resultados da análise de variância (ANOVA) para temperatura interna do frango, mostrando que o modelo é adequado (não foi significativa a falta de ajuste da regressão) com valor de $\mathrm{r}^{2}=0,8216$. Também foi observado que o tempo tem efeito significativo sobre a temperatura interna do produto $(\mathrm{p}<0,02)$, para as faixas de temperaturas e tempos estudados. Esses resultados diferem do reportado por WÄHLBY, SKJÖLDEBRAND \& JUNKER [14] que mencionaram que a temperatura interna é altamente dependente da temperatura do ar. Nos modelos físicos de transferência de calor, tal como a Segunda Lei de Fourier, a temperatura num ponto estacionário dentro do alimento depende da temperatura do meio de aquecimento, do tempo e a resistência à transferência de calor pelo alimento [3], que nosso modelo, por ser empírico, não foi capaz de explicar.

\section{2 - Rendimento}

Na Tabela 3 se aprecia o ANOVA para o rendimento do frango, onde se observa que o modelo é bom, sem evidência de falta de ajuste, com valor de $r^{2}=0,9345$.

Os principais efeitos que influenciaram sobre essa resposta foram o tempo e a temperatura $(\mathrm{p}<0,01)$.

WÄHLBY, SKJÖLDEBRAND \& JUNKER [14] mencionaram que, durante o cozimento de carne suína em um forno de injeção direta de vapor, a perda de água 
pela carne foi muito influenciada pela temperatura do ar, sendo pouco dependente do tempo de processo, e as maiores perdas estavam associadas com a utilização de altas temperaturas e curtos tempos.

TABELA 2. Análise de variância para a variável resposta temperatura interna do frango

\begin{tabular}{cccccc}
\hline Efeitos & $\begin{array}{c}\text { Graus de } \\
\text { liberdade }\end{array}$ & $\begin{array}{c}\text { Soma dos } \\
\text { quadrados }\end{array}$ & $\begin{array}{c}\text { Quadrados } \\
\text { médios }\end{array}$ & Relação $-\mathrm{F}$ & Valor - $\mathrm{P}$ \\
\hline $\mathrm{t}$ (tempo) & 1 & 43,2016 & 43,2016 & 12,88 & 0,0157 \\
$\mathrm{~T}$ (temperatura) & 1 & 6,8266 & 6,8266 & 2,04 & 0,2130 \\
$\mathrm{t}^{2}$ & 1 & 7,6652 & 7,6652 & 2,29 & 0,1910 \\
$\mathrm{~T}^{2}$ & 1 & 9,3004 & 9,3004 & 2,77 & 0,1567 \\
$\mathrm{~T}$ x T & 1 & 10,2400 & 10,2400 & 3,05 & 0,1410 \\
Falta de ajuste & 3 & 4,4810 & 1,4936 & 0,24 & 0,8618 \\
Erro puro & 2 & 12,2866 & 6,1433 & & \\
Total & 10 & 94,0018 & & & \\
$\mathrm{r}^{2}$ & 0,8216 & & & & \\
\hline
\end{tabular}

TABELA 3. Análise de variância para a variável resposta rendimento de frango

\begin{tabular}{cccccc}
\hline Efeitos & $\begin{array}{c}\text { Graus de } \\
\text { liberdade }\end{array}$ & $\begin{array}{c}\text { Soma dos } \\
\text { quadrados }\end{array}$ & $\begin{array}{c}\text { Quadrados } \\
\text { médios }\end{array}$ & Relação $-\mathrm{F}$ & Valor -P \\
\hline $\mathrm{t}$ (tempo) & 1 & 1,6016 & 1,6016 & 48,98 & 0,0009 \\
$\mathrm{~T}$ (temperatura) & 1 & 0,6666 & 0,6666 & 20,39 & 0,0063 \\
$\mathrm{~T}^{2}$ & 1 & 0,0214 & 0,0214 & 0,66 & 0,4544 \\
$\mathrm{~T}^{2}$ & 1 & 0,0030 & 0,0030 & 0,09 & 0,7731 \\
$\mathrm{t} \times \mathrm{T}$ & 1 & 0,0400 & 0,0400 & 1,22 & 0,3191 \\
Falta de ajuste & 3 & 0,1368 & 0,0456 & 3,42 & 0,2344 \\
Erro puro & 2 & 0,0266 & 0,0133 & & \\
Total & 10 & 2,4963 & & & \\
$\mathrm{r}^{2}$ & 0,9345 & & & & \\
\hline
\end{tabular}

Os coeficientes dos dados experimentais para os modelos de segunda ordem, das respostas estudadas são apresentados na Tabela 4.

TABELA 4. Coeficientes de regressão para o polinômio de segunda ordem para as variáveis de resposta

\begin{tabular}{ccc}
\hline Coeficientes & Temperatura interna & Rendimento \\
\hline$\beta_{0}$ & 82,984211 & 97,836842 \\
$\beta_{1}$ & 1,066667 & $-0,333333$ \\
$\beta_{2}$ & 2,683333 & $-0,516667$ \\
$\beta_{11}$ & $-2,310526$ & 0,057895 \\
$\beta_{22}$ & 1,739474 & $-0,100000$ \\
$\beta_{12}$ & 1,600000 & $-0,092105$ \\
\hline
\end{tabular}

\section{3 - Otimização}

Nas Figuras 1 e 2 estão apresentados os gráficos de superfície de resposta para a temperatura interna e rendimento dos filés de frango empanado. A partir dessas figuras foram plotadas as linhas de nivel ou de contorno (Figuras $3 e 4$ ), que permitem apreciar as diferentes condições de tempo e temperatura e obter diferentes valores de resposta.

A localização das condições ótimas de cozimento puderam ser encontradas a partir do método analítico, onde os pontos estacionários foram obtidos a partir da análise canônica (MYERS, 1971 citado por QUINTERO-
RAMOS et al. [10]). Para a temperatura interna do produto os resultados encontrados foram $209,9^{\circ} \mathrm{C}$, para a temperatura do ar, e um tempo de 3,6 minutos. Para o rendimento, as condições foram de $211,5^{\circ} \mathrm{C}$ e $2,9 \mathrm{minu}-$ tos. Em razão das respostas obtidas não estarem localizadas na mesma zona, optou-se por uma otimização gráfica, para determinar as condições ótimas de cozimento de acordo com o método recomendado por FLOROS \& CHINNAN [6] que consiste em sobrepor os gráficos de linhas de contorno das variáveis em estudo (Figuras 3 e 4). Através das curvas de nivel estimou-se que as melhores condições de processo correspondem à superfície compreendida entre as temperaturas do ar do forno de 206 e $209^{\circ} \mathrm{C}$ e tempos de cozimento entre 3,5 a 3,6 minutos, que correspondem as respostas de temperaturas internas compreendidas entre 82,7 e $83,6^{\circ} \mathrm{C}$ e rendimentos na faixa de 98,2 e $98,5 \%$.

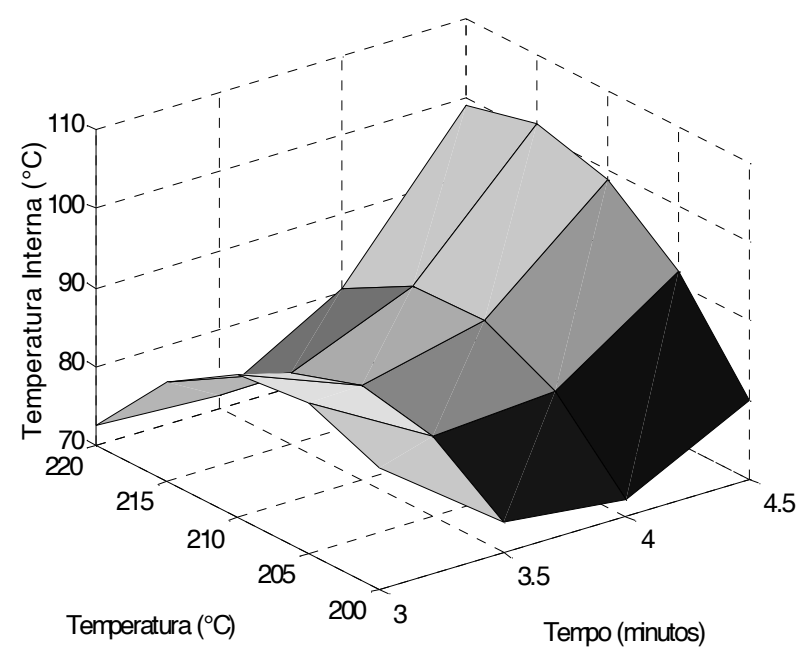

FIGURA 1. Superfície de resposta para a temperatura interna em função da temperatura e do tempo

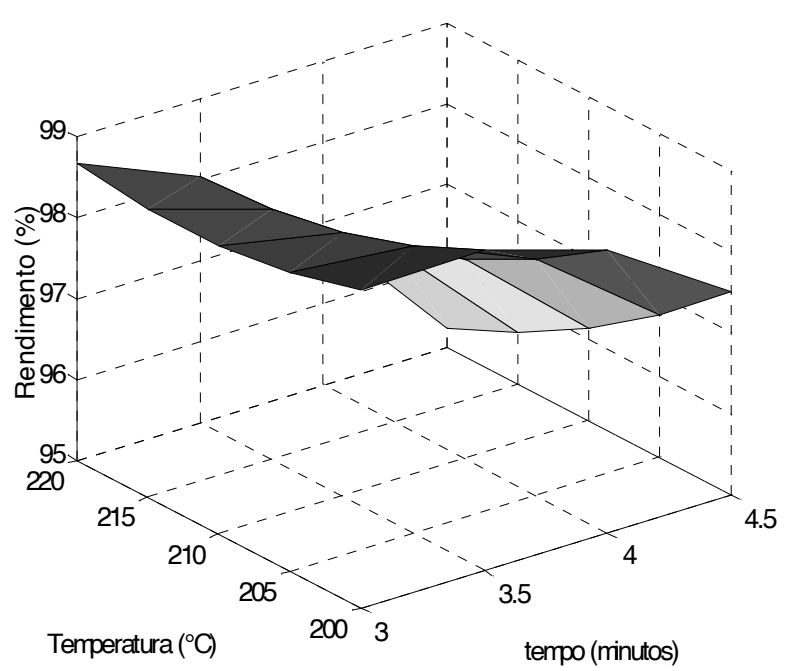

FIGURA 2. Superfície de resposta para o rendimento em função da temperatura e do tempo 


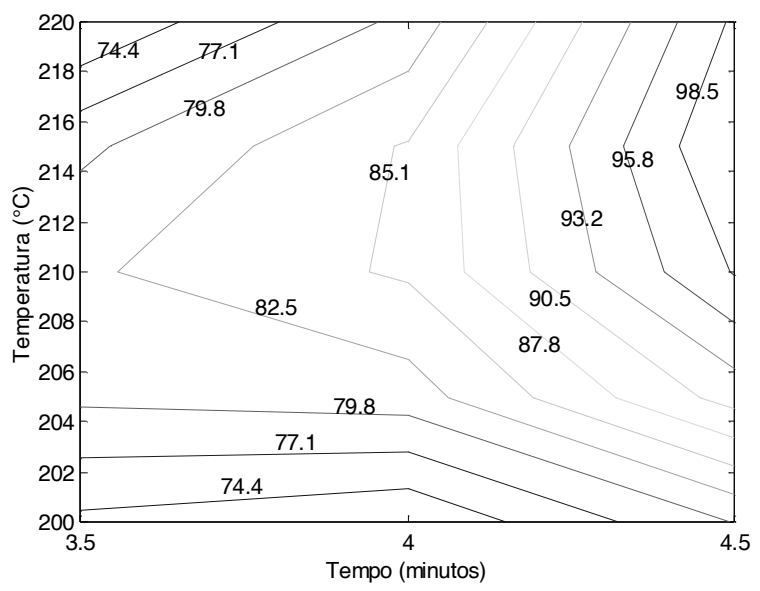

FIGURA 3. Curvas de nível para a temperatura interna em função da temperatura e do tempo

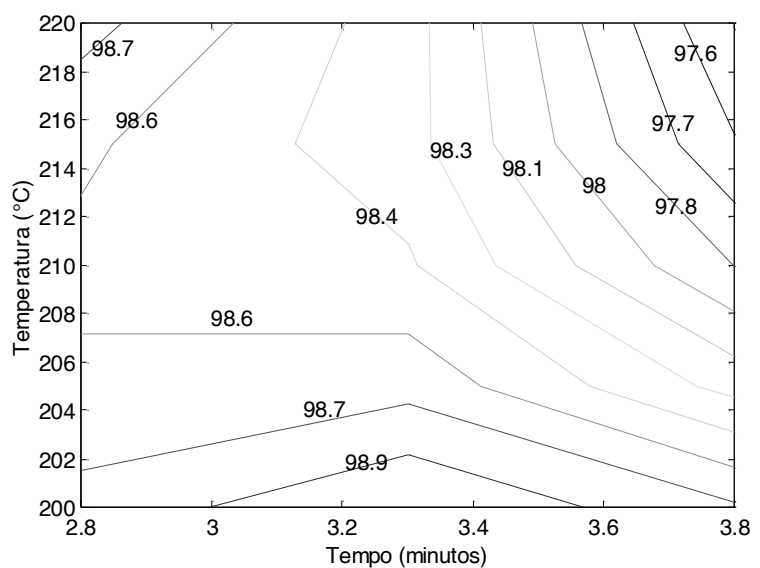

FIGURA 4. Curvas de nível para o rendimento em função da temperatura e do tempo

\section{4 - CONCLUSÕES}

Os modelos estatísticos de superfície de resposta obtidos foram apropriados, ajustando-se razoavelmente com respeito aos dados experimentais, que, em conexão com a técnica gráfica, ajudaram a localizar as condições ótimas de processo. Verificou-se que as melhores condições de cozimento correspondem à superfície compreendida entre as temperaturas do ar do forno de 206 e $209^{\circ} \mathrm{C}$ e tempos de cozimento entre 3,5 a 3,6 minutos, que correspondem as respostas de temperaturas internas 82,7 e $83,6^{\circ} \mathrm{C}$ e rendimentos na faixa de 98,2 e $98,5 \%$.

\section{5 - REFERÊNCIAS BIBLIOGRÁFICAS}

[1] ANG, C.Y.W.; HUANG, Y.W. Color changes of chicken leg patties due to end-point temperature, packaging and refrigerated storage. Journal of Food Science, v. 59, n. 1, p. 26-29, 1994.

[2] BERRY, B.W. Fat level, high temperature cooking and degree of doneness affect sensory, chemical and physical properties of beef patties. Journal of Food Science, v. 59, n. 1, p. 10-14, 1994.

[3] CHANG, H.C.; CARPENTER, J.A.; TOLEDO, R. Modeling coupled heat transfer during oven roasting of unstuffed turkeys. Journal of Food Science, v. 63, n.2, p. 257 $261,1998$.

[4] CHEN, H.; MARKS, B.P.; MURPHY, R.Y. Modeling coupled heat and mass transfer for convection cooking of chicken patties. Journal of Food Science, v. 42, n. 3, p. 139-146, 1999.

[5] CHRISTENSEN, M.; PURSLOW, P.P.; LARSEN M.L. The effect of cooking temperature on mechanical properties of whole meat, single muscle fibres and perimysial connective tissue. Meat Science, v. 55, p. 301-307, 2000.

[6] FLOROS, J.D.; CHINNAN, M.S. Computer graphicsassisted optimization for product and process development. Food Technology, v. 42, n. 2, p. 72-78, 1988.

[7] HANSELMAN, D.; LITTLEFIELD, B. MATlAB 5: Guia do Usuário. São Paulo: Makron Books, 1999. 413 p.

[8] HULTIN, H.O. Características del tejido muscular. In: FENNEMA, O. R. Química de los alimentos. Zaragoza: Acribia, S. A., 1985. Cap. 12, p. 815-888.

[9] PEARSON, A.M.; GILleTt, T.A. Processed meats. New York: Chapman \& Hall, 1996. 448 p.

[10] QUINTERO-RAMOS, A.; BOURNE, M.C.; BARNARD, J.; ANZALDÚA-MORALES, A. Optimization of low temperature blanching of frozen jalapeño pepper (Capsicum annuum) using response surface methodology. Journal of Food Science, v. 63, n. 3, p. 519-522, 1998.

[11] RESURRECCION, A.V.A. Cookery of muscle food. In: KINSAMN, D. M.; KOTULA, A. W.; BREIDENSTEIN, B. C. Muscle foods: meat, poultry and seafood technology. New York: Chapman \& Hall, 1994. p. 406429.

[12] SAS. Statistical Analysis System, versão 6.08. The SAS Institute, Cary, N. C., 1992.

[13] SHERIDAN, P.S.; SHILTON, N.C. Analysis of yield while cooking beefburguer patties using far infrared radiation. Journal of Food Engineering, v. 51, p. 3-11, 2002.

[14] WÄHLBY, U; SKJÖLDEBRAND, C.; JUNKER, E. Impact of impingement on cooking time and food quality. Journal of Food Engineering, v. 43, n. 3, p. 179$187,2000$. 